\title{
Mitral Balloon Valvotomy
}

National Cancer Institute

\section{Source}

National Cancer Institute. Mitral Balloon Valvotomy. NCI Thesaurus. Code C99987.

Dilation of the valve between the left atrium and the left ventricle of the heart by inflating a balloon. (ACC) 